\title{
Gutzwiller theory of band magnetism in LaOFeAs
}

\author{
Tobias Schickling, ${ }^{1}$ Florian Gebhard, ${ }^{1}$ Jörg Bünemann, ${ }^{2}$ Lilia Boeri, ${ }^{3}$ Ole K. Andersen, ${ }^{3}$ and Werner Weber ${ }^{4}$ \\ ${ }^{1}$ Department of Physics and Materials Science Center, Philipps Universität, 35032 Marburg, Germany \\ ${ }^{2}$ Institut für Physik, BTU Cottbus, P.O. Box 101344, 03013 Cottbus, Germany \\ ${ }^{3}$ Max-Planck-Institute for Solid-State Research, Heisenbergstr. 1, 70569 Stuttgart, Germany \\ ${ }^{4}$ Theoretische Physik II, Technische Universität Dortmund, Otto-Hahn-Str. 4, 44227 Dortmund, Germany
}

(Dated: November 5, 2018)

\begin{abstract}
We use the Gutzwiller variational theory to calculate the ground-state phase diagram and quasiparticle bands of LaOFeAs. The Fe $3 d-\mathrm{As} 4 p$ Wannier-orbital basis obtained from density-functional theory defines the band part of our eight-band Hubbard model. The full atomic interaction between the electrons in the iron orbitals is parameterized by the Hubbard interaction $U$ and an average Hund's-rule interaction $J$. We reproduce the experimentally observed small ordered magnetic moment over a large region of $(U, J)$ parameter space. The magnetically ordered phase is a stripe spin-density wave of quasi-particles.
\end{abstract}

PACS numbers: 71.10.Fd, 71.20.Be, 71.27.+a

$\mathrm{LaOFeAs}$ is the first high-temperature Fe-based superconductor [1]. Its crystal structure is made of alternating layers of $(\mathrm{LaO})^{+}$and $(\mathrm{FeAs})^{-}$. Below $T_{\mathrm{s}}=150 \mathrm{~K}$, the material undergoes a structural phase transition from tetragonal to orthorhombic, followed by the onset of a stripe spin-density wave (SDW) order in the FeAs layers with wave vector $\mathbf{q}=(0, \pi)$. The values of the ordered moments vary from $m=0.3 \mu_{\mathrm{B}}$ to $0.8 \mu_{\mathrm{B}}[2-4]$. Under pressure or upon doping, the electrons in the FeAs layers become superconducting at $T_{\mathrm{c}}=56 \mathrm{~K}[5,6]$. The presence of Fe-pnictogen or chalcogen layers and the proximity of magnetism and superconductivity with fairly high $T_{\mathrm{c}}$ are common to almost all the iron-based superconductors known to date, and place these compounds in the class of unconventional superconductors like the cuprates.

However, the cuprates and pnictides show important differences. In contrast to the cuprates, the normal phase of the pnictides is always metallic; in fact, densityfunctional theory (DFT) calculations provide a qualitatively correct description of the electronic structure and Fermi surface (FS) of the paramagnetic (PM) phase [7, 8], and reproduce the correct magnetic structure of the SDW state [9]. However, sizable renormalizations of the DFT quasi-particle band masses are observed in optics, angular-resolved photo-emission spectroscopy (ARPES), and de-Haas-van-Alphen measurements [5]. Moreover, the values of the ordered moments calculated by DFT in the SDW phase, $m_{\mathrm{DFT}} \approx 1.8 \mu_{\mathrm{B}}$ or higher, are much larger than the experimentally observed values [7].

This discrepancy has lead to an intense ongoing debate on the nature of magnetism (localized vs. itinerant), and on the mechanism of suppression of the magnetic moment (long-range spin fluctuations vs. strong local electronic correlations). Adopting the correlated-electron viewpoint [10, 11], one faces the problem that very few theoretical methods are available for the description of itinerant magnetism in multi-band systems.
In this work, we employ the Gutzwiller variational theory $(\mathrm{GT})$ which describes the ground state and quasiparticle excitations of multi-band Fermi liquids to study the magnetic phase diagram of LaOFeAs; GT treats the full atomic interactions and is numerically much less involved than, e.g., Dynamical Mean-Field Theory (DMFT) 10 12]. This enables us to resolve the small energy differences between the PM and SDW phases in the pnictides. We shall find that the striped SDW in LaOFeAs has a small ordered moment over a large region of $(U, J)$ parameter space. This small-moment phase can be understood as the band magnetism of correlated quasi-particles.

We investigate the two-dimensional eight-band Hubbard model

$$
\hat{H}=\hat{H}_{0}+\hat{H}_{\mathrm{C}}=\sum_{i, j ; b, b^{\prime} ; \sigma} t_{i, j}^{b, b^{\prime}} \hat{c}_{i, b, \sigma}^{\dagger} \hat{c}_{j, b^{\prime}, \sigma}+\sum_{i} \hat{H}_{\mathrm{C}, i},
$$

where $\hat{H}_{0}$ describes the on-site energies and hopping integrals of electrons with spin $\sigma=\uparrow, \downarrow$ between Fe3d-As $4 p$ Wannier orbitals $b, b^{\prime}$ on sites $i, j$ (NMTO) [8]. The corresponding DFT bandstructure is shown in Fig. 1. We have neglected the interlayer hoppings and have used the glide plane to unfold to the Brillouin zone which contains only one formula unit per cell [8].

The local Hamiltonian $\hat{H}_{\mathrm{C}, i}$ describes the Coulomb interaction between the $d$-electrons. We use the Hubbard interaction $U$ and an averaged Hund's-rule interaction $J$ to derive the Racah coefficients $A, B, C$ with the atomic ratio $C / B=4$ which parameterize our multi-band interaction [13]. In the following, until in Figs. 4 and 5 we give the results for a characteristic set of values $U=8 \mathrm{eV}$ and $J=0.6 \mathrm{eV}[(U, J)=(8,0.6) \mathrm{eV}]$. This set, for which $J=0.075 U$, gives an ordered moment of $m=0.74 \mu_{\mathrm{B}}$, intermediate between two recent experimental values.

We fix the average number of $3 d$-electrons per Fe and of the $4 p$-electrons per As at their PM DFT-values, $n_{d}=$ 7.47 and $n_{p}=4.53$, respectively [8]. The polar-covalent 

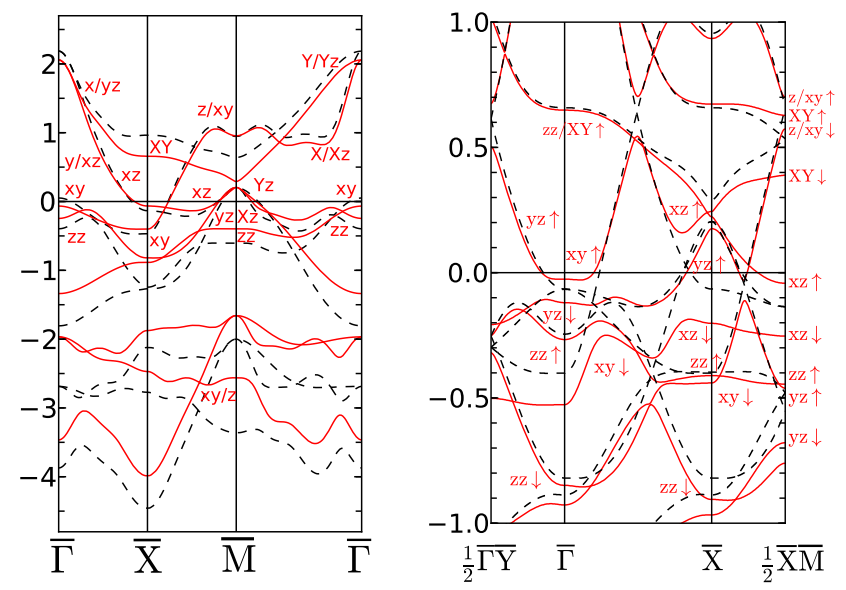

FIG. 1. (Color online) Fe3d-As $4 p$ bandstructure of LaOFeAs along the high-symmetry lines of the $2 \mathrm{D}$ Brillouin zone containing one formula unit per cell. Left: DFT [8] (dashed) and GT (solid) in the PM phase for $(U, J)=(8,0.6) \mathrm{eV}$. The bands are lined up at the Fermi level $(E=0)$, energies on the ordinate are in $\mathrm{eV}$, the DFT bandwidth is $W_{\mathrm{DFT}}=6.5 \mathrm{eV}$. Right: GT bands for $(U, J)=(8,0.6) \mathrm{eV}$ in the PM (dashed) and SDW (solid) phases in the Brillouin zone folded along the line $\frac{1}{2} \overline{\Gamma Y}-\frac{1}{2} \overline{\mathrm{XM}}$. High symmetry points and the directions $x$, $y$ and $X, Y$ are defined in Fig. 2 Dominant orbital and spin characters are indicated. Spin- $\uparrow$ are minority and spin- $\downarrow$ are majority spin states. For strongly covalent bands, $z / x y$ means more $z$ and less $x y$ character and vice versa [8].

bonding between Fe and As thus transfers 1.47 electrons from As to $\mathrm{Fe}$ compared with the ionic picture $\left(d^{6} p^{6}\right)$ implicit in the often used five-band model [14]. It has been shown previously [13] that the ionic picture does not lead to a satisfactory description of the magnetically ordered phase of LaOFeAs within GT. We do not fix the occupations of each of the five $3 d$ orbitals but there is little charge flow between them as a function of $(U, J)$ for the parameter values considered.

The true ground state of $\hat{H}$ in (1) is approximated by the Gutzwiller variational wave function

$$
\left|\Psi_{\mathrm{G}}\right\rangle=\hat{P}_{\mathrm{G}}\left|\Psi_{0}\right\rangle=\prod_{i} \hat{P}_{i}\left|\Psi_{0}\right\rangle
$$

where $\left|\Psi_{0}\right\rangle$ is a product state of Bloch orbitals, and the local Gutzwiller correlator is defined as

$$
\hat{P}_{i}=\sum_{\Gamma} \lambda_{\Gamma}|\Gamma\rangle_{i i}\langle\Gamma|
$$

Variational parameters $\lambda_{\Gamma}$ have been introduced for each multiplet state $|\Gamma\rangle_{i}$ on the iron sites, i.e., the eigenstates of $\hat{H}_{\mathrm{C}, i}$. Note that $\left|\Psi_{0}\right\rangle$ is also a variational object which we determine from the minimization of the variational energy functional resulting from the wave functions (2).

As shown in Refs. [15, 16], expectation values can be evaluated without further approximations in the limit of infinite spatial dimensions. The energy functional derived in this limit is then used as an approximation for finite-dimensional systems. In addition, GT provides the Landau-Gutzwiller quasi-particle bandstructure for comparison with ARPES data [17, 18].

We begin with the hight-temperature PM phase and present the quasi-particle bandstructure in Fig. 11 The electron-electron interaction between the $d$-electrons is seen to reduce the overall bandwidth. Filled bands with a large $3 d$ character [8] are shifted upwards in energy and empty, $3 d$-like bands downwards. Also, the three lowest bands are effected since of the 6 electrons in these As $p$ like bands, 1.82 are Fe $d$.
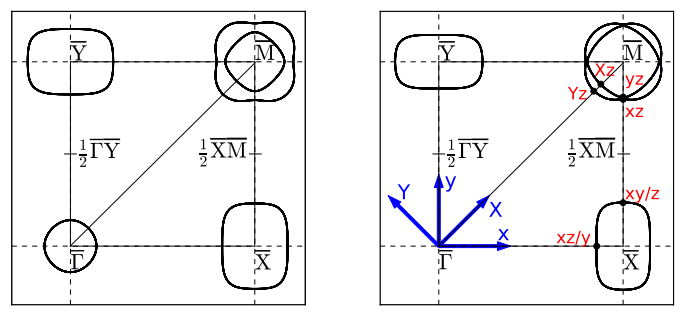

FIG. 2. (Color online) PM Fermi surface. Left: DFT; Right: GT for $(U, J)=(8,0.6) \mathrm{eV}$. The lower and upper case coordinate systems used to designate the orbitals are shown.

In Fig. 2 we show the FS calculated with DFT and GT for $(U, J)=(8,0.6) \mathrm{eV}$ for the PM phase. In GT the correlations shift the $x y$ band down by $130 \mathrm{meV}$ and thereby empty the $\bar{\Gamma}$-centered hole pocket. The concomitant lowering of the Fermi level narrows the $\overline{\mathrm{X}}$ and $\overline{\mathrm{Y}}$-centered electron pockets and makes the inner $\overline{\mathrm{M}}$-centered hole pocket expand. The lowering of the empty $X Y / Y z$ like band near $\overline{\mathrm{M}}$ steepens the lower, $Y z$-like conduction band [8] and thereby shrinks the outer $\overline{\mathrm{M}}$-centered hole pocket along $\bar{M}-\bar{\Gamma}$. The small size of these effects confirms the conventional wisdom that DFT bands provide a reasonable description of the FS. On the other hand, the agreement of the DFT FS with experiment does not indicate that the electron-electron interactions are weak.

The correlation-induced mass renormalization on the FS is defined as the renormalization of the Fermi velocity, $m^{*} / m=v_{\mathrm{F}, \mathrm{DFT}} / v_{\mathrm{F}}$. It depends on the orbital character of the band states involved. For pure $d$-bands, the renormalization is $q_{d} \approx 1.5$ for $(U, J)=(8,0.6) \mathrm{eV}$ and grows by $3 \%$ per eV increase of $U$. The mass enhancement decreases towards unity with increasing $p$ character. Also band-shifts influence the effective masses on the Fermi surface. For the six points where the Fermi surface cuts the high-symmetry lines in Fig. 2, $\left(m^{*} / m\right)_{n}=1.6,1.2,1.3,1.3,1.4,1.3$ for the orbitals $n=$ $x z / y, x y / z, x z, y z, X z, Y z$. Note that the $x z / y$-like DFT band in $\bar{\Gamma}-\overline{\mathrm{X}}$ direction has a strong curvature in the vicinity of $\overline{\mathrm{X}}$ and, as the correlation-induced downwards shift of the $x y$ band drags the Fermi level along, it is placed lower in the $x z / y$ band. This increases $\left(m^{*} / m\right)_{x z / y}$ beyond the renormalization factor $q_{d}$. 

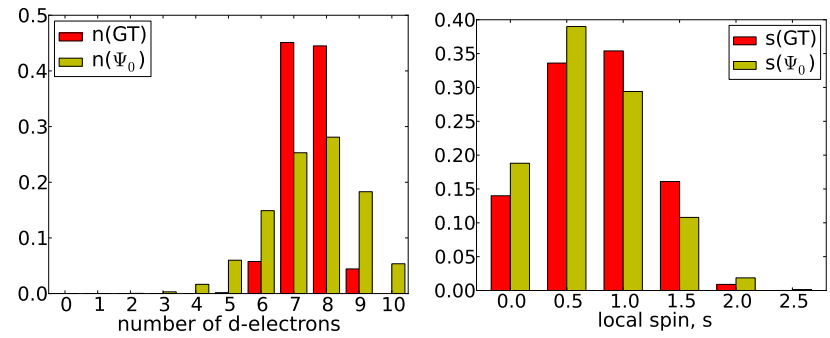

FIG. 3. (Color online) Local $d$-charge distribution $p(n)$ (left) and local spin distribution $p(s)$ (right) for the PM GT with $(U, J)=(8,0.6) \mathrm{eV}$ and for the PM single-particle wave function $\left|\Psi_{0}\right\rangle$.

For $(U, J)=(8,0.6) \mathrm{eV}$, the dominant $3 d$-configurations are $3 d^{7}$ and $3 d^{8}$ in the optimized PM Gutzwiller wave function $\left|\Psi_{\mathrm{G}}^{\mathrm{opt}}\right\rangle$. Occupancies with $n_{d}=6$ or $n_{d}=9$ electrons are rare, and all other charge states are essentially forbidden, see Fig. 3. The absence of large charge fluctuations is typical for correlated electron systems. The probability distribution function $p(s)$ for finding local spins with size $0 \leq s \leq 5 / 2$ in $\left|\Psi_{\mathrm{G}}^{\mathrm{opt}}\right\rangle$ is very similar to the distribution in the single-particle product state $\left|\Psi_{0}\right\rangle$. In GT, the correlation enhancement of the local spin moment is small. This shows that the system is far from the local-moment situation where we would solely find atoms with Hund's-rule spins $s=3 / 2$ for $3 d^{7}$ and $s=1$ for $3 d^{8}$. From the expectation values for the local spin, $\left\langle\left(\hat{\mathbf{S}}_{i}\right)^{2}\right\rangle=\sum_{s} p(s) s(s+1)$, we find $\left\langle\left(\hat{\mathbf{S}}_{i}\right)^{2}\right\rangle=1.62$ and $\left\langle\left(\hat{\mathbf{S}}_{i}\right)^{2}\right\rangle_{0}=1.41$ for $\left|\Psi_{\mathrm{G}}^{\mathrm{opt}}\right\rangle$ and $\left|\Psi_{0}\right\rangle$, respectively, considerably smaller than $\left\langle\left(\hat{\mathbf{S}}_{i}\right)^{2}\right\rangle_{s=3 / 2}=15 / 4$ for $s=3 / 2$ and $\left\langle\left(\hat{\mathbf{S}}_{i}\right)^{2}\right\rangle_{s=1}=2$ for $s=1$.

Now, we turn to the SDW. The local charge and spin distributions shown in Fig. 3 change only slightly from the PM to the SDW phase, as also seen in DMFT [11]. The exchange splitting, $\Delta$, is within $10 \%$ the same for all five $d$-orbitals and amounts to $\Delta=0.37 \mathrm{eV}$ for $(U, J)=(8,0.6) \mathrm{eV}$. The $d_{y z}, d_{x y}, d_{3 z^{2}-r^{2}}$, and $d_{x^{2}-y^{2}}$ orbitals contribute almost equally to the magnetization, which is $m=0.74 \mu_{\mathrm{B}}$ for $(U, J)=(8,0.6) \mathrm{eV}$, whereas the $d_{x z}$ orbital, whose band hardly disperses in the antiferromagnetic direction, is twice as polarized as the others. Its exchange splitting is $10 \%$ larger.

On the right-hand side of Fig. 1, we compare the quasiparticle bandstructures of the PM and SDW phases for $(U, J)=(8,0.6) \mathrm{eV}$. Where the PM $E(\mathbf{k})$-bands cross the $E(\mathbf{k}+\mathbf{q})$ bands $(\mathbf{q}=[\bar{\Gamma} \bar{Y}]=[\overline{\mathrm{X}} \overline{\mathrm{M}}])$ they split approximately by $\Delta$ times the overlap of their $d$-orbital characters [8]. This leads to a noticeable rearrangement of the bands. In GT also band-shifts are permitted. Nevertheless, the GT quasi-particle bands and FS in the SDW phase are similar to the results of Stoner theory using the PM DFT bands from $\hat{H}_{0}$ in (1) and the exchangeinteraction constant $I_{\text {Stoner }}$ adjusted to give the same moment [8].

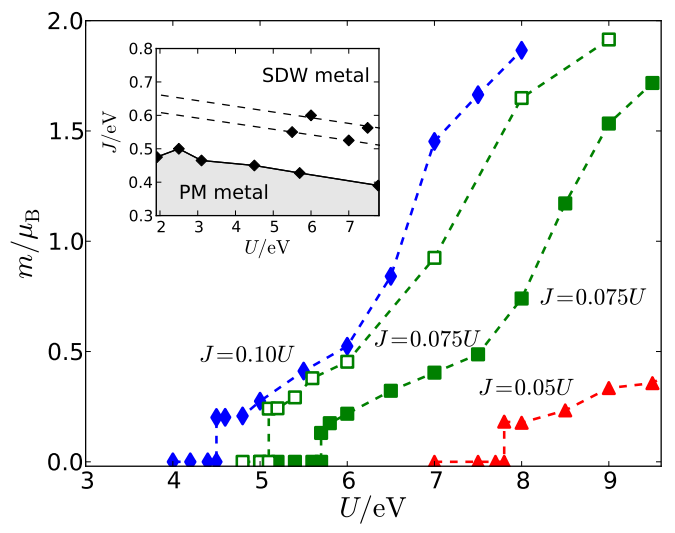

FIG. 4. (Color online) GT ordered magnetic moment as a function of $U$ for various values of $J / U$. Our characteristic point $(U, J)=(8,0.6) \mathrm{eV}$ is a filled square. Full symbols: full atomic Hamiltonian; open symbols: restricted atomic Hamiltonian with density-density interactions only. Inset: Ground-state phase diagram as a function of $U$ and $J$. In the SDW metal, we also show lines for constant magnetization, $m=0.4 \mu_{\mathrm{B}}$ and $m=0.5 \mu_{\mathrm{B}}$, respectively.

The dependence of the ordered magnetic moment on the atomic parameters $(U, J)$ in GT is shown in Fig. 4. We find a remarkably broad region in the $(U, J)$ parameter space with a small ordered moment $m \lesssim 0.8 \mu_{\mathrm{B}}$, in contrast to a description based on six Fe electrons in five orbitals [13, 14]. As for the five-band model [13], the PM metal is the ground state for not too large values of the Hund's-rule coupling $J$, in contrast to Hartree-Fock theory where symmetry breaking occurs for small $U$.

The atomic Hamiltonian is frequently approximated by density-density interactions only, e.g. in DMFT [10, 12] in order to keep the numerical problems under control. One of the magnetization curves in Fig. 4 compares the GT result of this approximation with the GT for the full Hamiltonian. We see that the former approximation largely overestimates the magnetization.

The inset of Fig. 4 4 shows that the onset of the SDW requires a finite $J \geq J_{\mathrm{c}}(U) \approx I_{\mathrm{c}}-0.017 U$ with $I_{\mathrm{c}}=0.52 \mathrm{eV}$ $(U \geq 3 \mathrm{eV})$. Moreover, in the SDW region, the moment is to a very good approximation a function of merely the linear combination $J+0.017 U$. Thus, we then can identify $I(U, J)=J+0.017 U$ as the effective low-energy scale for the magnetic excitations of our eight-band model in GT. That not only the onset but the entire GT magnetization collapses onto one curve when plotted as a function of $I$ is seen in Fig. 5. This shows that the Hund's-rule coupling and not the Hubbard-repulsion is the controlling parameter for the magnetism in this system. This may explain why DMFT studies of LaOFeAs obtain a small ordered moment using quite different $U$-values 10 12].

Using the PM DFT Hamiltonian $\hat{H}_{0}$, eq. (1), we calculate the magnetization, $m(\Delta)$, as a function of an external staggered field $\Delta$. The resulting $\Delta / m(\Delta) \equiv \chi_{\mathrm{DFT}}^{-1}$ 


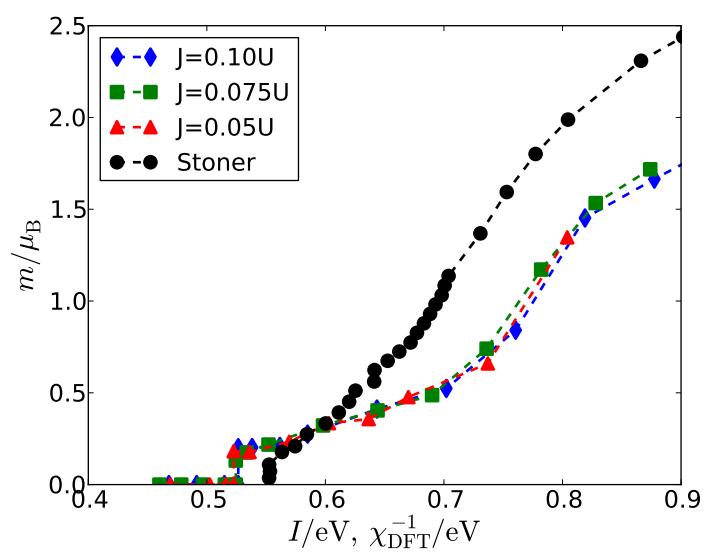

FIG. 5. (Color online) Ordered moment as a function of $I=$ $J+0.017 U$ for various values of $J / U$ in GT (diamonds), and the inverse susceptibility in DFT $\chi_{\mathrm{DFT}}^{-1}=\Delta / m(\Delta)$ (dots). The ordered moment given by spin-DFT (GGA) is reproduced by $I_{\mathrm{DFT}}=0.82 \mathrm{eV}[8]$.

is a pure bandstructure function, which in Stoner theory provides the selfconsistent magnetization as the solution of the equation $\chi_{\mathrm{DFT}}^{-1}(m)=I_{\text {Stoner }}[8]$. This function is plotted in Fig. 5 . As seen from the size of the magnetization jump, the transition from the PM to the SDW state in GT is driven by the same band mechanism. Details differ due to different underlying PM bandstructures.

The existence of a sharp 'nose' at small moments is caused by the nesting of the hole and electron sheets with common $x z$ character, while the strong increase of the moment, once $m \gtrsim 0.75 \mu_{\mathrm{B}}$ in GT, occurs when the $x y$ exchange splitting is so large that the spin- $\uparrow$ minority band empties at $\bar{\Gamma}=\overline{\mathrm{Y}}$, and thereby starts to contribute to the magnetization. At this point also the $z z$ magnetization picks up in GT. This is mainly due to $z z$ hybridization of the empty $X Y$ minority band near $\bar{Y}$. Nevertheless, over the entire parameter range, $m_{x z}>m_{x y}>m_{z z}>m_{X Y}>$ $m_{y z}$ in GT. For DFT the moment increases faster after the nose and $m_{x z}=m_{x y}>m_{z z}>m_{X Y}>m_{y z}$. The first difference is due to the steeper bands and the latter to the slightly different band positions. Roughly speaking, the GT of correlated quasi-particles supports the Stoner picture of band magnetism for the small-moment phase in LaOFeAs, provided that the DFT exchangecorrelation kernel is substituted by a weak, low-energy Stoner parameter.

The correlated band picture can be further scrutinized from the analysis of the GT susceptibility $\chi_{\mathrm{GT}}=$ $\Delta_{\mathrm{GT}} / m\left(\Delta_{\mathrm{GT}}\right)$ (not shown). Following Landau Fermiliquid theory, we write it as $\chi_{\mathrm{GT}} / \chi_{\mathrm{DFT}}=q_{d} /\left(1+F_{0}^{\mathrm{a}}\right)$ where $q_{d}$ is the average mass enhancement factor and $F_{0}^{\text {a }}$ is a Landau parameter. The enhancement of the susceptibility is mostly due to $q_{d}(U, J)$, i.e., $\chi_{\mathrm{GT}} / q_{d}$ depends on the interaction through $I(m)$ only, thus explaining why the three GT curves in Fig. [5 can be rescaled to a uni- versal curve even though their mass enhancements are very different. For all $m$ we find that $\chi_{\mathrm{GT}} /\left(q_{d} \chi_{\mathrm{DFT}}\right)=$ $1 /\left(1+F_{0}^{\mathrm{a}}(m)\right) \approx 0.8$. A small positive value $F_{0}^{\mathrm{a}}(m) \approx 0.2$ reflects the tendency to anti-ferromagnetic spin alignment. The applicability of Fermi-liquid theory supports the DFT picture of band magnetism in LaOFeAs.

In this work, we have employed Gutzwiller theory to calculate the quasi-particle bands for a two-dimensional eight-band Hubbard model for the valence electrons in the iron-arsenic planes of LaOFeAs. In a large region of the $(U, J)$ parameter space, we find a spin-density wave ground state of quasi-particles with a small magnetic moment $m \lesssim 0.8 \mu_{\mathrm{B}}$, as observed experimentally. When charge fluctuations are suppressed by the Hubbard- $U$, the Hund'-rule coupling $J$ is the effective low-energy scale which drives the transition to metallic band magnetism.

In view of the qualitatively correct DFT description of the paramagnetic and also the spin-density wave phases (allowing for a renormalization of $I_{\mathrm{DFT}} \sim 0.8 \mathrm{eV}$ to $\left.I_{\text {Stoner }} \sim 0.6 \mathrm{eV}\right)$, the DFT bandstructure provides a reasonable starting point for investigations of the iron-based superconductors. The Gutzwiller theory offers a microscopic Fermi-liquid description for the correlation corrections due to the electron-electron interaction.

This work was supported in part by the Deutsche Forschungsgemeinschaft (SPP 1458 and Bo-3536/1).

[1] Y. Kamihara, T. Watanabe, M. Hirano, and H. Hosono. J. Am. Chem. Soc., 130:3296, 2008.

[2] C. de la Cruz et al. Nature (London), 453:899, 2008.

[3] N. Qureshi et al. Phys. Rev. B, 82:184521, 2010.

[4] H. F. Li et al. Phys. Rev. B, 82:064409, 2010.

[5] J. Paglione and R. L. Greene. Nature physics, 6:645, 2010.

[6] D. C. Johnston. Adv. in physics, 59:803, 2010.

[7] D. J. Singh and M.-H. Du. Phys. Rev. Lett., 100:237003, 2008.

[8] O. K. Andersen and L. Boeri. Ann. Physik (Berlin), 523:8-50, 2011.

[9] I. I. Mazin et al. Phys. Rev. Lett., 101:057003, 2008.

[10] M. Aichhorn et al. Phys. Rev. B, 80:085101, 2009.

[11] Z. P. Yin, K. Haule, and G. Kotliar. Nature physics, 7:294, 2011.

[12] P. Hansmann et al. Phys. Rev. Lett., 104:197002, 2010.

[13] T. Schickling, F. Gebhard, and J. Bünemann. Phys. Rev. Lett., 106:146402, 2011.

[14] S. Graser, T. Maier, P. Hirschfeld, and D. Scalapino. New Journal of Physics, 11:025016, 2009.

[15] J. Bünemann, W. Weber, and F. Gebhard. Phys. Rev. B, 57:6896, 1998.

[16] J. Bünemann, F. Gebhard, and W. Weber. In A. Narlikar, editor, Frontiers in Magnetic Materials, pages 117151. Springer, Berlin, 2005.

[17] J. Bünemann, F. Gebhard, and R. Thul. Phys. Rev. B, 67:75103, 2003.

[18] A. Hofmann et al. Phys. Rev. Lett., 102:187204, 2009. 